\title{
$\alpha$-Synuclein Gene May Interact with Environmental Factors in Increasing Risk of Parkinson's Disease
}

\author{
Nicole M. Gatto ${ }^{a}$ Shannon L. Rhodes ${ }^{a}$ Angelika D. Manthripragada ${ }^{a}$ \\ Jeff Bronstein ${ }^{c}$ Myles Cockburn ${ }^{d}$ Matt Farrer $^{\mathrm{e}}$ Beate Ritz ${ }^{\mathrm{a}-c}$ \\ Departments of a Epidemiology, ${ }^{\mathrm{b}}$ Environmental Health Sciences and ${ }^{\mathrm{C}}$ Neurology, University of California \\ Los Angeles, and d Department of Preventive Medicine, University of Southern California, Los Angeles, Calif., USA, \\ and ${ }^{\text {}}$ Department of Medical Genetics, University of British Columbia, Vancouver, B.C., Canada
}

\section{Key Words}

$\alpha$-Synuclein • Pesticides • Parkinson's disease · Smoking •

Age of onset $\cdot$ Paraquat $\cdot$ Interaction $\cdot$ REP1

\begin{abstract}
Background: Although of great interest and suggested in prior reports, possible $\alpha$-synuclein (SNCA) gene-environment interactions have not been well investigated in humans. Methods: We used a population-based approach to examine whether the risk of Parkinson's disease (PD) depended on the combined presence of SNCA variations and two important environmental factors, pesticide exposures and smoking. Results/Conclusions: Similar to recent metaand pooled analyses, our data suggest a lower PD risk in subjects who were either homozygous or heterozygous for the SNCA REP1 259 genotype, and a higher risk in subjects who were either homozygous or heterozygous for the REP1 263 genotype, especially among subjects with an age of onset $\leq 68$ years. More importantly, while analyses of interactions were limited by small cell sizes, risk due to SNCA variations seemed to vary with pesticide exposure and smoking, especially in younger onset cases, suggesting an age-of-onset effect.

Copyright $\odot 2010$ S. Karger AG, Basel
\end{abstract}

\section{KARGER}

Fax +41613061234 E-Mail karger@karger.ch www.karger.com
() 2010 S. Karger AG, Basel

www.karger.com/ned

\section{Introduction}

Abnormal aggregation of the $\alpha$-synuclein protein, a major component of Lewy bodies and a hallmark of Parkinson's disease (PD), is believed to be a critical step in the molecular pathogenesis of the disease [1]. Multiplications of the $\alpha$-synuclein gene (SNCA) have been seen in familial PD [2], and functional data demonstrate that SNCA expression varies across different alleles of the dinucleotide repeat sequence (REP1) within the SNCA promoter [3]. Several single nucleotide polymorphisms (SNPs) and haplotypes in the SNCA promoter have also been shown to be associated with risk of sporadic PD [4], and increasing REP1 length is associated with an increased risk of PD $[5,6]$, as well as possibly with a decrease in age of PD onset [5]. Multiple lines of evidence point to pesticide exposures as possible contributors to the pathogenesis of idiopathic PD; many epidemiological studies have separately reported associations between pesticide exposure in general, rural living, farming, and the development of PD [7]. We have previously shown that a mbient exposure to a combination of paraquat and maneb pesticides increases the risk of developing PD (especially for younger onset cases) [8], that exposure to pesticides through consumption of potentially contaminated well
Prof. Beate Ritz, MD, PhD

Schools of Public Health and Medicine, UCLA

650 Charles E. Young Drive, Box 951772

Los Angeles, CA 900951772 (USA)

Tel. +1 310206 7458, Fax +1 310206 6039,E-Mail britz@ucla.edu 
water increases PD risk [9], and that genetic variability in the dopamine transporter gene interacts with pesticide exposures to further increase PD risk [10]. $\alpha$-Synuclein transgenic mouse models demonstrate exacerbated neuropathology when exposed to paraquat [11] or maneb and paraquat combined [12]. While it is generally recognized that multiple insults likely contribute to the occurrence of PD in humans, only one published epidemiologic study to date investigated possible SNCA-pesticide interactions, and this study relied on retrospective self-reports of pesticide exposure [13]. In addition, results from a recent study suggest that smoking may be an important modifying factor of the SNCA REP1-PD association [14].

In contrast to previous studies, our population-based case-control study of PD estimated long-term pesticide exposure from agricultural applications in rural counties of California's Central Valley using a geographic information system approach that integrates unique statemandated pesticide use reports (PUR) and land use data. Here, we examine whether the presence of SNCA variations interact with two recognized environmental risk factors, pesticide exposure and smoking, to influence the risk of developing PD.

\section{Materials and Methods}

Included in the following analyses were 333 incident idiopathic PD cases from three rural California counties (Kern, Tulare, Fresno) enrolled in the Parkinson's Environment Gene (PEG) Study [15] who were recruited within 3 years of first diagnosis and for whom our UCLA movement disorder specialists confirmed PD diagnoses; 336 population controls were randomly selected from the same tri-county area using Medicare or residential parcels records. All subjects provided informed consent, and the study was approved by the UCLA Institutional Review Board. Trained interviewers blinded to case/control status conducted structured telephone interviews to obtain demographics, lifetime address histories, and other risk factor information including smoking behavior from study participants.

We employed a validated geographic information systembased exposure assessment tool to estimate ambient pesticide exposure within $500 \mathrm{~m}$ of subjects' homes resulting from pesticide applications to agricultural crops [16]. We combined geocoded lifetime address data of participants, historical PUR data collected by the California Department of Pesticide Regulation since 1974, and land use data (to determine locations of pesticide application more precisely $[17,18])$ to estimate ambient exposure for all historical residential addresses reported from 1974 to 1999 , the period prior to PD onset and covered by the PUR data. We summed pesticide applications (pounds per acre applied within a 500 -meter buffer zone) at residences across the 26 -year period to calculate the cumulative total for ambient paraquat exposure for each subject. Exposure to paraquat at or above the median value in the control population was considered high, and below the me- dian including no exposure was considered low/no exposure. Genomic DNA was extracted from a blood or buccal sample provided by participants and SNCA REP1 allele-length variants [ $\leq 259$ bp ('short'), 261 bp, $\geq 263$ bp ('long')] were genotyped using PCR with forward and reverse primers. We also genotyped DNA for two SNCA SNPs previously shown to be associated with PD [6]: rs356165 (in coding region) and rs2583988 (in promoter region) using $\mathrm{ABI}$ Taqman ${ }^{\circledR}$. Hardy-Weinberg equilibrium was verified for the two SNPs in PD cases and control subjects using $\chi^{2}$ tests.

Following the work of previous investigators $[5,6]$, we assumed a dominant inheritance model, and used multivariable unconditional logistic regression methods to assess main effects of REP1 allele-length variant genotypes. We calculated odds ratios (ORs) and $95 \%$ confidence intervals (95\% CIs) comparing the 259 or 263 REP1 variants to other genotypes adjusting for self-reported age (continuous), sex, education ( $<12$ years, 12 years, $>12$ years), race/ ethnicity (White, non-White), family history of PD (first-degree relative with PD: yes/no) and smoking (never, former, current). We used stratified analyses to evaluate associations within subgroups of sex, smoking (ever, never), family history of PD, and age at diagnosis to distinguish younger versus older onset $\mathrm{PD}$ ( $\leq 68$ years, $>68$ years) using the median age in cases as a cut-point so as to maximize our statistical efficiency and as previously used in the meta-analysis [6] (we did not have enough very young onset cases, i.e. age $\leq 50$ years, to explore a younger age cut-off used by Kay et al. [5]). To further examine gene-environment interactions between paraquat exposure and REP1 genetic variants, we conducted stratified analyses by age at PD onset. All analyses used SAS version 9.1 (SAS Institute Inc., Cary, N.C., USA).

\section{Results}

Study participants were predominantly White $(\mathrm{n}=$ $583,87.1 \%)$, over the age of 65 years $(\mathrm{n}=443,66.2 \%)$, and without a family history of PD $(n=532,79.5 \%)$. Cases were slightly older than controls, more likely to be male, had completed fewer years of education, and were more likely to have never smoked cigarettes. We did not find any associations between genotypes of the two SNCA SNPs and PD in our study (data not shown).

Similar to the previous meta- and pooled analyses [5, 6], we found that subjects who were either homozygous or heterozygous for the REP1 259 genotype exhibited a slightly lower risk of developing PD compared to carriers of all other REP1 genotypes, while subjects who were either homozygous or heterozygous for the REP1 263 genotype exhibited a slightly increased risk of PD (table 1). While our effect estimates were similar in magnitude to those reported in the meta-analysis for Caucasians, due to our smaller sample size, the $95 \%$ CIs for our estimates included the null value of 1 . Also similar to prior studies, we observed a stronger association among those with younger onset PD ( $\leq 68$ years of age in our study). We 
Table 1. Results from adjusted logistic regression models

\begin{tabular}{lccc}
\hline Sample or stratum & Cases/controls & OR (95\% CI) & \\
\cline { 3 - 4 } & & $259 / 259$ or 259/X vs. X/X & $263 / 263$ or 263/X vs. X/X \\
\hline Maraganore et al. [6] meta-analysis; all subjects & $2,686 / 2,454$ & $0.85(0.76-0.96)$ & $1.44(1.21-1.70)$ \\
Kay et al. [5] pooled analysis; all subjects & $1,779 / 2,079$ & $0.83(0.72-0.95)$ & $1.23(1.01-1.51)$ \\
PEG study [15]; all subjects & $333 / 336$ & $0.93(0.66-1.29)$ & $1.26(0.78-2.04)$ \\
$\quad$ Caucasians only & $290 / 293$ & $0.93(0.65-1.31)$ & $1.50(0.87-2.56)$ \\
Family history & & & \\
$\quad$ Negative & $285 / 247$ & $0.86(0.60-1.22)$ & $1.43(0.85-2.39)$ \\
$\quad$ Positive & $48 / 33$ & $1.73(0.64-4.69)$ & $0.48(0.10-2.25)$ \\
Male & $184 / 169$ & $0.81(0.51-1.27)$ & $1.14(0.58-2.25)$ \\
Female & $149 / 167$ & $1.10(0.67-1.81)$ & $1.25(0.61-2.58)$ \\
Age of onset & & & \\
$\quad \leq 68$ years & $144 / 168$ & $0.95(0.57-1.58)$ & $2.26(1.04-4.91)$ \\
$\quad>68$ years & $189 / 168$ & $0.87(0.55-1.37)$ & $0.87(0.46-1.64)$ \\
Never smokers & $125 / 100$ & $0.91(0.50-1.65)$ & $2.81(1.10-7.16)$ \\
Ever smokers & $208 / 236$ & $0.97(0.65-1.45)$ & $0.88(0.49-1.58)$
\end{tabular}

$\mathrm{X} / \mathrm{X}$ represents any other genotype. OR adjusted for age (continuous), sex, education $(<12,12$ or $>12$ years), race (White/non-White), smoking status (former, current or never), and family history (positive/negative in a first-degree relative) when not a stratifying variable.

Table 2. Results from adjusted logistic regression models stratified by paraquat exposure and age of onset

\begin{tabular}{|c|c|c|c|c|c|c|c|c|}
\hline \multirow[t]{2}{*}{ Genotype } & \multicolumn{8}{|c|}{ Paraquat exposure - cases/controls, OR $(95 \% \text { CI })^{1}$} \\
\hline & \multicolumn{4}{|l|}{ low/no } & \multicolumn{4}{|l|}{ high } \\
\hline \multirow{6}{*}{$\begin{array}{l}259 / 259 \text { or } 259 / X \\
\text { vs. } X / X \\
263 / 263 \text { or } 263 / X \\
\text { vs. } X / X\end{array}$} & $106 / 131$ & \multirow[t]{2}{*}{$0.77(0.51-1.15)$} & & & $72 / 49$ & \multicolumn{3}{|l|}{$1.35(0.74-2.46)$} \\
\hline & $104 / 105$ & & & & $51 / 51$ & & & \\
\hline & $31 / 29$ & \multirow{2}{*}{\multicolumn{3}{|c|}{$1.22(0.68-2.18)$}} & $18 / 13$ & \multirow{2}{*}{\multicolumn{3}{|c|}{$1.45(0.59-3.59)$}} \\
\hline & $179 / 207$ & & & & $105 / 87$ & & & \\
\hline & \multicolumn{8}{|c|}{ Age of onset - cases/controls, OR $(95 \% \mathrm{CI})^{2}$} \\
\hline & \multicolumn{2}{|l|}{$\leq 68$ years } & \multicolumn{2}{|c|}{$>68$ years } & \multicolumn{2}{|c|}{$\leq 68$ years } & \multicolumn{2}{|c|}{$>68$ years } \\
\hline $259 / 259$ or $259 / \mathrm{X}$ & $41 / 66$ & $0.67(0.36-1.24)$ & $65 / 65$ & $0.89(0.53-1.51)$ & $37 / 26$ & $1.96(0.81-4.76)$ & $35 / 23$ & $0.87(0.37-2.06)$ \\
\hline vs. X/X & $47 / 50$ & & $57 / 55$ & & $19 / 26$ & & $32 / 25$ & \\
\hline $263 / 263$ or $263 / \mathrm{X}$ & $15 / 13$ & $1.68(0.69-4.08)$ & $16 / 16$ & $0.89(0.41-1.93)$ & $9 / 4$ & $3.15(0.74-13.37)$ & 9/9 & $0.84(0.27-2.62)$ \\
\hline vs. $\mathrm{X} / \mathrm{X}$ & $73 / 103$ & & $106 / 104$ & & $47 / 48$ & & $58 / 39$ & \\
\hline
\end{tabular}

$\mathrm{X} / \mathrm{X}$ represents any other genotype.

${ }^{1}$ Adjusted for age (continuous), sex, education ( $<12,12$ or $>12$ years), race (White/non-White), smoking status (former, current or never), and family history (positive/negative in a first-degree relative).

${ }^{2}$ Adjusted for age (continuous), sex, and smoking status (former, current or never).

observed little indication of either a gender-specific or family history-specific effect, although our study has too few subjects with a positive family history to be informative. Consistent with prior work [14], we observed an increase in risk of PD (OR 2.81, 95\% CI 1.10-7.16) for never smokers carrying the 263 allele repeat, while a history of smoking seemed to reverse the direction of the effect associated with this repeat ( $\mathrm{p}$-interaction $=0.06$ ).

When combined with a high level of paraquat exposure, the normally protective SNCA 259 allele was unexpectedly associated with an increased risk of PD (p-interaction $=0.10)($ table 2$)$. When stratifying by both para- 
quat exposure level and age of PD onset, our results suggest that the association is due to an increased risk in subjects with disease onset at or prior to age 68 years $(\mathrm{OR}=3.15,95 \% \mathrm{CI} 0.74-13.37)$. In fact, for subjects with disease onset after age 68 years, our data suggest little to no effect for either the SNCA 263 repeat allele or paraquat exposure (table 2). Exploration of joint effects for exposure to both maneb and paraquat and variations in the SNCA gene were precluded by the small numbers of subjects available for these analyses.

\section{Discussion}

In summary, our data agree with previous meta- and pooled analysis results suggesting that a shorter repeat length SNCA REP1 genotype (indicated by $\leq 259$ in our study) may protect against $\mathrm{PD}$, while the longer repeat length REP1 genotype ( $\geq 263)$ confers an increased risk for PD, particularly for younger onset cases. Our data strongly support a previous observation of a smokingSNCA interaction [14], such that never smokers who also carry at least one copy of the 263 repeat length genotype are at an increased risk of developing PD. In our population, the majority of subjects were exposed to agricultural pesticides, and our data suggest that the protective effect of the shorter REP1 repeat length polymorphism may be lost in the presence of pesticide exposure, and that the longer REP1 genotype may increase susceptibility to pesticide exposures and contribute to an increased PD risk in younger onset cases. Our analyses are based on small numbers of subjects categorized as younger onset who carried the 263 repeat polymorphism and who were highly exposed to paraquat ( 9 cases and 4 controls with onset $\leq 68$ years). Thus, our finding should be re-examined in larger studies that include a greater number of pesticideexposed subjects who also carry rare SNCA REP1 alleles. A strength of our study compared to previous work examining SNCA-pesticide interactions [13] is the availability of accurate long-term historical pesticide use data. Our results speak not only to the importance of valid exposure data, but also to the necessity of large well-characterized samples of exposed populations for informative investigations of gene-environment interactions. Our findings highlight the multi-factorial nature of PD etiology, as demonstrated here particularly for younger onset cases for whom exposure to high levels of pesticides seems to modify genetic susceptibility, such that environmental insults may contribute to an even greater PD risk among risk allele carriers, and diminish or negate advantages in carriers of protective alleles. This cautions against the naive interpretation of genetic analyses across populations with heterogeneous exposures - that the effect of an allele seen in one population might not be found in another due to the interplay of such genes with a complex environment.

\section{Acknowledgement and Funding}

This study was supported by grants T32ES01545, ES10544, U54ES12078, and 5P30ES07048 from the NIEHS; NS038367 from the NINDS; PC051037 from the DOD; and funding from the Michael J. Fox Foundation. We thank Amit Joshi for his assistance with creating haplotypes.

\section{References}

1 Lashuel HA, Petre BM, Wall J, Simon M, Nowak RJ, Walz T, Lansbury PT Jr: Alphasynuclein, especially the Parkinson's disease-associated mutants, forms pore-like annular and tubular protofibrils. J Mol Biol 2002;322:1089-1102.

-2 Nishioka K, Hayashi S, Farrer MJ, Singleton $A B$, Yoshino H, Imai H, Kitami T, Sato K, Kuroda R, Tomiyama $\mathrm{H}$, Mizoguchi $\mathrm{K}, \mathrm{Mu}-$ rata $\mathrm{M}$, Toda $\mathrm{T}$, Imoto I, Inazawa J, Mizuno Y, Hattori N: Clinical heterogeneity of alphasynuclein gene duplication in Parkinson's disease. Ann Neurol 2006;59:298-309.

3 Chiba-Falek O, Touchman JW, Nussbaum RL: Functional analysis of intra-allelic variation at NACP-Repl in the alpha-synuclein gene. Hum Genet 2003;113:426-431.

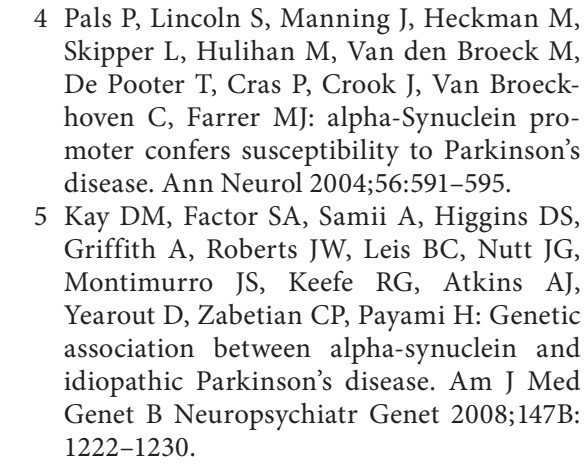
1222-1230.

\footnotetext{
6 Maraganore DM, de Andrade M, Elbaz A, Farrer MJ, Ioannidis JP, Krüger R, Rocca WA, Schneider NK, Lesnick TG, Lincoln SJ, Hulihan MM, Aasly JO, Ashizawa T, Chartier-Harlin MC, Checkoway H, Ferrarese C, Hadjigeorgiou G, Hattori N, Kawakami H, Lambert JC, Lynch T, Mellick GD, Papapetropoulos S, Parsian A, Quattrone A, Riess O, Tan EK, Van Broeckhoven C, Genetic Epidemiology of Parkinson's Disease (GEO-PD) Consortium: Collaborative analysis of alpha-synuclein gene promoter variability and Parkinson disease. JAMA 2006;296:661670.

7 Priyadarshi A, Khuder SA, Schaub EA, Priyadarshi SS: Environmental risk factors and Parkinson's disease: a metaanalysis. Environ Res 2001;86:122-127.
} 
8 Costello S, Cockburn M, Bronstein J, Zhang $\mathrm{X}$, Ritz B: Parkinson's disease and residential exposure to maneb and paraquat from agricultural applications in the central valley of California. Am J Epidemiol 2009;169:919926.

9 Gatto NM, Cockburn M, Bronstein J, Manthripragada AD, Ritz B: Well-water consumption and Parkinson's disease in rural California. Environ Health Perspect 2009; 117:1912-1918.

- 10 Ritz BR, Manthripragada AD, Costello S, Lincoln SJ, Farrer MJ, Cockburn M, Bronstein J: Dopamine transporter genetic variants and pesticides in Parkinson's disease. Environ Health Perspect 2009;117:964-969.

-11 Fernagut PO, Hutson CB, Fleming SM, Tetreaut NA, Salcedo J, Masliah E, Chesselet MF: Behavioral and histopathological consequences of paraquat intoxication in mice: effects of alpha-synuclein over-expression. Synapse 2007;61:991-1001.
12 Norris EH, Uryu K, Leight S, Giasson BI, Trojanowski JQ, Lee VM: Pesticide exposure exacerbates alpha-synucleinopathy in an A53T transgenic mouse model. Am J Pathol 2007;170:658-666.

13 Brighina L, Frigerio R, Schneider NK, Lesnick TG, de Andrade M, Cunningham JM, Farrer MJ, Lincoln SJ, Checkoway H, Rocca WA, Maraganore DM: Alpha-synuclein, pesticides, and Parkinson disease: a case-control study. Neurology 2008;70:1461-1469.

14 McCulloch CC, Kay DM, Factor SA, Samii A, Nutt JG, Higgins DS, Griffith A, Roberts JW, Leis BC, Montimurro JS, Zabetian CP, Payami H: Exploring gene-environment interactions in Parkinson's disease. Hum Genet 2008;123:257-265.
15 Kang GA, Bronstein JM, Masterman DL, Redelings M, Crum JA, Ritz B: Clinical characteristics in early Parkinson's disease in a central California population-based study. Mov Disord 2005;20:1133-1142.

16 Ritz B, Costello S: Geographic model and biomarker-derived measures of pesticide exposure and Parkinson's disease. Ann NY Acad Sci 2006;1076:378-387.

17 Goldberg DW, Zhang X, Marusek JC, Wilson JP, Ritz B, Cockburn MG: Development of an automated pesticide exposure analyst for the California's Central Valley. Proc Urban Regional Info Syst Assoc GIS Public Health Conf, New Orleans, 2007.

18 Rull RP, Ritz B: Historical pesticide exposure in California using pesticide use reports and land-use surveys: an assessment of misclassification error and bias. Environ Health Perspect 2003;111:1582-1589. 